\title{
SYMMETRIC GRAPHS WITH PROJECTIVE SUBCONSTITUENTS
}

\author{
RICHARD WEISS
}

\begin{abstract}
Let $\Gamma$ be a finite, undirected, connected graph and $G$ a subgroup of $\operatorname{aut}(\Gamma)$ acting transitively on the vertex set of $\Gamma$ such that the stabilizer $G(x)$ in $G$ of a vertex $x$ contains a normal subgroup which induces a permutation group on the set of vertices adjacent to $x$ isomorphic to $\operatorname{PSL}(n, q)$ with $n \geqslant 3$. A bound for $|G(x)|$ depending only on $n$ and $q$ is shown to exist under certain conditions.
\end{abstract}

Let $\Gamma$ be an undirected graph and $G$ a subgroup of aut $(\Gamma)$ acting transitively on the vertex set of $\Gamma$. Let $x$ be an arbitrary vertex of $\Gamma$. We denote by $\Gamma(x)$ the set of vertices adjacent to $x$ and by $G(x)^{\Gamma(x)}$ the permutation group induced by the stabilizer $G(x)$ of $x$ in $G$ on $\Gamma(x) ; G(x)^{\Gamma(x)}$ is called the subconstituent of the pair $(\Gamma, G)$. For each $i \in \mathbf{N}$ let $G_{i}(x)=\{a \in G(x) \mid a \in$ $G(y)$ if $\partial(x, y) \leqslant i\}$ where $\partial(x, y)$ denotes the distance between $x$ and $y$. Let $s \in \mathbf{N}$. An $s$-path is a sequence $\left(x_{0}, x_{1}, \ldots, x_{s}\right)$ of $s+1$ vertices $x_{i}$ such that $x_{i-1} \in \Gamma\left(x_{i}\right)$ if $1 \leqslant i \leqslant s$ and $x_{i-2} \neq x_{i}$ if $2 \leqslant i \leqslant s$. For each $s$-path $\left(x_{0}, \ldots, x_{s}\right)$ let $G\left(x_{0}, \ldots, x_{s}\right)=G\left(x_{0}\right) \cap \cdots \cap G\left(x_{s}\right)$ and $G_{i}\left(x_{0}, \ldots, x_{s}\right)$ $=G_{i}\left(x_{0}\right) \cap \cdots \cap G_{i}\left(x_{s}\right)$ for each $i \in \mathbf{N}$. The graph $\Gamma$ is called $(G, s)$ transitive if $G$ acts transitively on the set of $s$-paths but not on the set of $(s+1)$-paths in $\Gamma$.

In [6], Tutte showed that if $\Gamma$ is finite, connected, trivalent and $(G, s)$ transitive with $s \geqslant 1$ then $s \leqslant 5$ and $|G(x)|=3 \cdot 2^{s-1}$. Subsequently considerable attention has been given to the problem of finding a similar result for graphs of arbitrary valency; see, for instance, [1], [3] and [9]. In the present paper we discuss the case that $P S L(n, q) \leqslant G(x)^{\Gamma(x)} \leqslant P \Gamma L(n, q)$ with $n \geqslant 3$. In [8], it was shown that $s \leqslant 3$ in this case; however, it seems much more difficult to find a bound for $|G(x)|$ when $\operatorname{PSL}(n, q) \leqslant G(x)^{\Gamma(x)}$ $\leqslant P \Gamma L(n, q)$ with $n \geqslant 3$. We prove the following partial result:

THEOREM. Let $n$ be a natural number greater than two and $q$ a power of some prime $p$. Let $\Gamma$ be a finite, undirected, connected graph and suppose that aut $(\Gamma)$ contains a subgroup $G$ such that $\Gamma$ is $(G, 3)$-transitive with $\operatorname{PSL}(n, q)$ $\leqslant G(x)^{\Gamma(x)} \leqslant P \Gamma L(n, q)$ for every vertex $x$ (where PSL $(n, q)$ and $P \Gamma L(n, q)$ are to be considered as acting on the set of points of the projective space $P G(n-1, q)$ in the usual fashion). Then $\left|G_{1}(x, y)\right| \mid q^{(n-1)^{2}}$ for every edge $\{x, y\}$ if $p \geqslant 5$.

Received by the editors November 21, 1977.

AMS (MOS) subject classifications (1970). Primary 05C25, 20B25, 05B25; Secondary 20 G40. Key words and phrases. Symmetric graph, projective space, metasymplectic space. 
Before turning to the proof, we note two important examples. First, let $q$ be an arbitrary prime power, $n \geqslant 3, G$ the group of all collineations and correlations of $P G(2 n-1, q)$ and $\Gamma$ the bipartite graph whose vertices are the $(n-2)$ - and $(n-1)$-dimensional subspaces of $P G(2 n-1, q)$ where two such subspaces are defined to be adjacent when one of them contains the other. Let $\{x, y\}$ be an edge of $\Gamma$. Then $\Gamma$ is connected, $(G, 3)$-transitive, $G(x)^{\Gamma(x)}=P \Gamma L(n, q)$ and $\left|G_{1}(x, y)\right|=q^{(n-1)^{2}}$. Thus the bound for $\left|G_{1}(x, y)\right|$ given in the theorem is the best possible one.

When $q$ is even, $\left|G_{1}(x, y)\right| \mid q^{(n-1)^{2}}$ need not hold, at least when $n=3$. To see this, let $S$ be the metasymplectic space associated with the group $F_{4}(q), q$ even (see [5, (10.13)]). Let $\Gamma$ be the bipartite graph whose vertices are the lines and planes of $S$ where a line $L$ is defined to be adjacent to a plane $P$ when $P$ contains $L$. Let $G$ be the group of all collineations and correlations of $S$. By [5, (5.10)], $G$ acts transitively on the vertex set of $\Gamma$. For each plane $P$ of $S$, $G(P)$ acts like $P \Gamma L(3, q)$ on the projective space consisting of $P$ together with the subspaces it contains. If $\{x, y\}$ is an edge of $\Gamma$, then $O_{2}(G(x, y))$ is isomorphic to a 2-Sylow group of $F_{4}(q)$ and thus $\left|G_{1}(x, y)\right|=q^{18}$.

To show that $\Gamma$ is $(G, 3)$-transitive, let $P_{1}$ and $P_{2}$ be planes of $S$ and $L_{1}$ and $L_{2}$ lines such that $\left(P_{1}, L_{1}, P_{2}, L_{2}\right)$ is a 3-path in $\Gamma$. Since $L_{1}$ and $L_{2}$ both lie in $P_{2}, L_{1} \cap L_{2} \neq \varnothing$; let $\alpha$ be the point of $S$ lying in $L_{1} \cap L_{2}$. Since $P_{1} \cap P_{2}$ contains $L_{1}$, there exists dually a symplectum $s$ of $S$ containing both $P_{1}$ and $P_{2}$. Let $\Delta$ be the flag complex of $S$. Let $C_{0}=\left\{\alpha, L_{1}, P_{1}, s\right\}, C_{1}=$ $\left\{\alpha, L_{1}, P_{2}, s\right\}$ and $C_{2}=\left\{\alpha, L_{2}, P_{2}, s\right\}$. Then $C_{0}, C_{1}$ and $C_{2}$ are chambers of $\Delta$ and $\left(C_{0}, C_{1}, C_{2}\right)$ is a minimal gallery. Since $\Delta$ is a building, there is an apartment $\Sigma$ of $\Delta$ containing both $C_{0}$ and $C_{2}$; by [5, (3.18)], $\Sigma$ contains $C_{1}$ too. If $\Pi$ is the subgraph of $\Gamma$ induced by the lines and planes of $S$ in $\Sigma$, then $\left(P_{1}\right.$, $\left.L_{1}, P_{2}, L_{2}\right)$ is a 3-path in $\Pi$. Let $\Phi$ be the bipartite graph whose vertices are the edges and triangles of the regular polytope $\{3,4,3\}$ (see [2, p. 149]) where an edge $E$ is defined to be adjacent to a triangle $T$ when $T$ contains $E$. Then $\Pi \cong \Phi$ and the stabilizer of $\Sigma$ in $G$ induces an automorphism group on $\Pi$ isomorphic to the symmetry group $[3,4,3]$ of $\{3,4,3\}$. For each edge $E$ of $\{3,4,3\}$, the stabilizer of $E$ in $[3,4,3]$ acts transitively on the set of twelve 3-paths of $\Phi$ beginning at $E$. Since $G$ acts transitively on the set of all apartments of $\Delta, \Gamma$ is in fact $(G, 3)$-transitive.

In the following lemma we list a few elementary properties of the projective groups which we will need in the proof of the theorem; verification is left to the reader:

LemMA. Let $H$ be a subgroup of $P \Gamma L(n, q)$ (with $n \geqslant 3$ ) containing $\operatorname{PSL}(n, q)$ considered as acting in the usual fashion on $P G(n-1, q), q$ a power of a prime $p \geqslant 5$. Let $X$ be the set of points of $P G(n-1, q), x \in X$ arbitrary and $Y$ the set of lines passing through $x$.

(i) If $1 \neq M \triangleleft K \varangle \triangleleft H(x)$, where $H(x)$ denotes the stabilizer of $x$ in $H$, and $K^{Y} \neq 1$ then $K^{Y} \geqslant \operatorname{PSL}(n-1, q)$ and $M \geqslant O_{p}(H(x))$. $\left|O_{p}(H(x))\right|=$ 
$q^{n-1}, O_{p}(H(x))^{Y}=1$ and $O_{p}(H(x))$ acts transitively on $g-\{x\}$ for every $g \in Y$. The order of the largest subgroup of $H(x)$ acting trivially on $Y$ divides $(q-1) q^{n-1}$.

(ii) If $\left\{x_{1}, \ldots, x_{n}\right\}$ is a frame of $P G(n-1, q)$ (i.e., a set of $n$ points no three of which are collinear), then $\left\langle O_{p}\left(H\left(x_{1}\right)\right), \ldots, O_{p}\left(H\left(x_{n}\right)\right)\right\rangle$ acts transitively on $X$.

We now begin the proof of the theorem. Let $\Gamma$ be an arbitrary graph fulfilling the hypotheses. By [7, (7.61)] there exist two vertices $u$ and $v$ with $\partial(u, v)=2$. If $|\Gamma(u) \cap \Gamma(v)| \geqslant 2$ then $\Gamma$ is a complete bipartite graph since $G$ acts transitively on the set of all 3-paths in $\Gamma$. Thus we may assume that $|\Gamma(u) \cap \Gamma(v)|=1$ whenever $\partial(u, v)=2$.

For every vertex $x$ of $\Gamma$ we denote by $\mathscr{P}(x)$ the projective space of dimension $n-1$ with point set $\Gamma(x)$ induced by $G(x)$. For each edge $\{x, y\}$ let $\mathscr{P}(x, y)$ be the projective space of dimension $n-2$ whose subspaces of dimension $m$ are the subspaces of $\mathscr{P}(x)$ of dimension $m+1$ containing $y$ $(0 \leqslant m \leqslant n-2)$. Let $P(x, y)$ be the set of points of $\mathscr{P}(x, y)$. For each element $a$ of $G(x, y)$ let $a_{x y}$ be the permutation that $a$ induces on $P(x, y)$.

Now let $\{x, y\}$ be an arbitrary edge of $\Gamma$. We claim that $G_{1}(x)^{P(y, x)}$ $\geqslant P S L(n-1, q)$. Suppose not. By the lemma, $G_{1}(x)^{P(y, x)}=1$ since $G_{1}(x) \triangleleft$ $G(x, y)$. We define a map $f: G(x, y)^{P(x, y)} \rightarrow G(x, y)^{P(y, x)}$ as follows: Given an arbitrary element $c \in G(x, y)^{P(x y)}$, pick an element $a \in G(x, y)$ with $a_{x y}=c$ and set $f(c)=a_{y x}$. Suppose that $b$ is a second element of $G(x, y)$ with $b_{x y}=c$. Let $K=\left\{d \in G(x, y) \mid d_{x y}=1\right\}$. If $a_{y x} \neq b_{y x}$ then $K$ acts nontrivially on $P(y, x)$ and thus, by the lemma, $K^{P(y, x)} \geqslant P S L(n-1, q)$ since $K \varangle G(x, y)$. Since $\left[K: G_{1}(x)\right.$ ], by the lemma, divides $(q-1) q^{n-1}$ but $|P S L(n-1, q)|$ does not, $G_{1}(x)$ acts nontrivially on $P(y, x)$. This contradicts the assumption. Thus $f$ is well defined. Since $f$ is clearly a homomorphism and invertible, $f$ is induced, according to [4], by a map $\varphi$ from $\mathscr{P}(x, y)$ to $\mathscr{P}(y, x)$ which is either a correlation or a collineation. Let $w \in \Gamma(x)-\{y\}$ and $\alpha \in P(x, y)$ with $w \in \alpha$ be arbitrary; we have $G(w, x, y) \leqslant G(\alpha, x, y)$ where $G(\alpha, x, y)$ denotes the stabilizer of $\alpha$ in $G(x, y)$. Let $\beta=\varphi(\alpha)$. $\beta$ is a subspace of $\mathscr{P}(y, x)$ of dimension 0 or $n-3$. Since $G(\alpha, x, y)=G(x, y, \beta)$, $G(w, x, y)$ acts intransitively on $\Gamma(y)-\{x\}$. This contradicts the hypothesis that $G$ acts transitively on the set of all 3-paths in $\Gamma$. We conclude that $G_{1}(x)^{P(y, x)} \geqslant P S L(n-1, q)$, as claimed.

Let $z$ be an arbitrary vertex in $\Gamma(y)-\{x\}$. According to [3, (2.3)], $\left|G_{1}(x, y)\right|$ is a prime power. Since $G_{1}(x, y) \leqslant G_{1}(y) \varangle G(y, z), G_{1}(x, y)^{P(z, y)}$ $=1$ and $\left|G_{1}(x, y)^{\Gamma(z)}\right|=1$ or $q^{n-1}$ by the lemma. If $G_{1}(x, y)^{\Gamma(z)}=1$ then $G_{1}(x, y)=G_{1}(y, z)$ and thus $G_{1}(x, y) \leqslant\langle G(x, y), G(y, z)\rangle=G(y)$. Let $d$ be an element of $G$ exchanging $x$ and $y$. Then $G_{1}(x, y) \varangle\langle G(y), d\rangle$. Since $\Gamma$ is connected, $\langle G(y), d\rangle=G$ and thus $G_{1}(x, y)=1$. Hence we may assume that $\left|G_{1}(x, y)^{\Gamma(z)}\right|=q^{n-1}$. In particular, $G_{1}(x, y)$ is a $p$-group; since $G_{1}(x, y)$ $\varangle G_{1}(x) \leqslant G(x)$, we have $G_{1}(x, y) \leqslant O_{p}(G(x))$ and thus $O_{p}(G(x)) \neq 1$. We 
define a map $f: G_{1}(y)^{P(x, y)} \rightarrow G_{1}(y)^{P(z, y)}$ as follows: Given an arbitrary element $c \in G_{1}(y)^{P(x, y)}$, pick an element $a \in G_{1}(y)$ with $a_{x y}=c$ and set $f(c)=a_{z y}$. It is easily seen, just as in the previous paragraph, that $f$ is well defined.

Suppose that $f$ is induced by a correlation from $\mathscr{P}(x, y)$ to $\mathscr{P}(z, y)$. Let $u \in \Gamma(y)-\{x\}, u \neq z$, and $\alpha \in P(x, y)$ be arbitrary. Then there exist copoints $A$ of $\mathscr{P}(z, y)$ and $B$ of $\mathscr{P}(u, y)$ such that $G_{1}(y) \cap G(x, \alpha)=G_{1}(y)$ $\cap G(z, A)=G_{1}(y) \cap G(u, B)$ where $G(x, \alpha)$ denotes the stabilizer of $\alpha$ in $G(x)$, etc. Similarly, however, there exists a point $\beta$ of $\mathscr{P}(u, y)$ such that $G_{1}(y) \cap G(z, A)=G_{1}(y) \cap G(u, \beta)$. Thus $G_{1}(y) \cap G(u, B)=G_{1}(y) \cap$ $G(u, \beta)$ although $G_{1}(y)^{P(u, y)} \geqslant P S L(n-1, q)$. With this contradiction we conclude that $f$ is induced by a collineation from $\mathcal{P}(x, y)$ to $\mathcal{P}(z, y)$.

Now let $v$ be an arbitrary vertex of $\Gamma$ and $g$ an arbitrary line of $\mathcal{P}(v)$. For each pair of points $u$ and $w$ in $\Gamma(v)$ there is a collineation $\varphi_{u w}: \mathscr{P}(u, v) \rightarrow$ $\mathcal{P}(w, v)$ such that $G_{1}(v) \cap G(u, \alpha)=G_{1}(v) \cap G\left(w, \varphi_{u w}(\alpha)\right)$ for every point $\alpha$ of $\mathcal{P}(u, v)$. For each point $u$ of $g$ choose a point $\alpha_{u}$ of $\mathscr{P}(u, v)$ in such a way that $\varphi_{u w}\left(\alpha_{u}\right)=\alpha_{w}$ for every two points $u$ and $w$ of $g$. Let $\pi=\left\{\alpha_{u} \mid u \in g\right\}$ and let $d(v, g, \pi)$ denote the subgraph of $\Gamma$ induced by $v$ together with the $q^{2}+2 q+1$ vertices which are points of $g$ or one of the $\alpha_{u}$ in $\pi$. The vertex $v$ will be called the center of $d(v, g, \pi)$. Let $D$ be the set of all such subgraphs $d(v, g, \pi), v$ an arbitrary vertex of $\Gamma, g$ an arbitrary line of $\mathscr{P}(v)$ and $\pi$ an arbitrary set $\left\{\alpha_{u} \in P(u, v) \mid u \in g\right\}$ fulfilling the above condition. Let $\Delta$ be the undirected graph with vertex set $D$ where two vertices $d\left(v_{1}, g_{1}, \pi_{1}\right)$ and $d\left(v_{2}, g_{2}, \pi_{2}\right)$ are defined to be adjacent when $v_{1} \in g_{2}, v_{2} \in g_{1}, g_{1} \in \pi_{2}$ and $g_{2} \in \pi_{1}$. The graph $\Delta$ is regular of valency $q+1$ and $G$ acts faithfully as a group of automorphisms of $\Delta$.

Note that each 3-path $(w, x, y, z)$ in $\Gamma$ determines a unique vertex $d(x, g, \pi)$ of $\Delta$ with center $x$ such that $w$ and $y \in g$ and $z \in \alpha_{y} \in \pi$; let $d_{w x y z}$ denote this vertex. The group $G_{1}(y) \cap G(x, g)$ stabilizes $d_{w x y z}$ and acts transitively on $g-\{y\}$. It follows that $P S L(2, q) \leqslant G(d)^{\Delta(d)} \stackrel{\sim}{\leqslant} P L(2, q)$ for every $d \in D$. Since $G$ acts transitively on the set of 3-paths of $\Gamma, G$ acts transitively on $D$. Thus there exists a $t \geqslant 2$ such that the graph $\Delta$ is $(G, t)$-transitive. In [10], it was shown that if $\Delta$ is an arbitrary finite $(G, t)$ transitive graph with $P S L(2, q) \leqslant G(d)^{\Delta(d)} \leqslant P \Gamma L(2, q)$ for every vertex $d$, then either $t \leqslant 4$ or $t=2 p+1$ with $2 \leqslant p \leqslant 3$.

Now let $\left\{x_{2}, x_{3}\right\}$ be an arbitrary edge of $\Gamma$. If $G_{2}\left(x_{2}\right)=G_{2}\left(x_{3}\right)$, then $G_{2}\left(x_{3}\right) \varangle\left\langle G\left(x_{2}\right), G\left(x_{3}\right)\right\rangle$; since $\Gamma$ is connected, $\left\langle G\left(x_{2}\right), G\left(x_{3}\right)\right\rangle$ acts transitively on the edge set of $\Gamma$ and so $G_{2}\left(x_{3}\right)=1$. Suppose that $G_{2}\left(x_{2}\right) \neq G_{2}\left(x_{3}\right)$. Then there exists a vertex $x_{1} \in \Gamma\left(x_{2}\right)$ such that $G_{2}\left(x_{3}\right) \nless G_{1}\left(x_{1}\right)$. There exist vertices $x_{0}, x_{4}, x_{5}$ and $x_{6}$ such that $\left(x_{0}, x_{1}, x_{2}, \ldots, x_{6}\right)$ is a 6-path in $\Gamma$ and $\left(d_{0123}, d_{1234}, d_{2345}, d_{3456}, d_{6543}\right)$, where $d_{i j k l}=d_{x_{1} x_{j} x_{k} x_{i}}$, is a 4-path in $\Delta$ which we denote by $W$. Let $\alpha$ be the point of $\mathcal{P}\left(x_{1}, x_{2}\right)$ containing $x_{0}$. We have $G_{2}\left(x_{3}\right) \leqslant G(W)$ since $G_{2}\left(x_{3}\right)^{P\left(x_{1}, x_{2}\right)} \leqslant G_{1}\left(x_{2}, x_{3}\right)^{P\left(x_{1}, x_{2}\right)}=1$ and $G_{2}\left(x_{3}\right)^{P\left(x_{5}, x_{4}\right)}$ 
$\leqslant G_{1}\left(x_{3}, x_{4}\right)^{P\left(x_{5}, x_{4}\right)}=1$. Since $G_{2}\left(x_{3}\right) \triangleleft G_{1}\left(x_{2}\right) \triangleleft G\left(x_{1}, x_{2}\right), G_{2}\left(x_{3}\right)$ acts transitively on $\alpha-\left\{x_{2}\right\}$ by the lemma. Thus $G(W)$ acts transitively on $\Delta\left(d_{0123}\right)-\left\{d_{1234}\right\}$. Since $t \geqslant 2, G$ contains an element mapping an arbitrary 2-path in $\Delta$ to $\left(d_{2345}, d_{1234}, d_{0123}\right)$; thus $t \geqslant 3$. Hence $G$ contains an element mapping an arbitrary 3-path to $\left(d_{3456}, d_{2345}, d_{1234}, d_{0123}\right)$; thus $t \geqslant 4$ and so $G$ contains an element mapping an arbitrary 4-path to $W$. Hence $t \geqslant 5$. Since $p \geqslant 5$ implies that $t \leqslant 4$, we conclude that $G_{2}\left(x_{3}\right)=1$ after all.

Finally, let $x$ be an arbitrary vertex and $\left\{x_{1}, \ldots, x_{n}\right\}$ a frame of $\mathcal{P}(x)$. Let $J=G_{1}\left(x_{1}\right) \cap \cdots \cap G_{1}\left(x_{n}\right)$. Since $\left|G_{1}\left(x_{1}, x\right)^{\Gamma\left(x_{i}\right)}\right|=q^{n-1}$ for $2 \leqslant i \leqslant n$, we have $|J| \geqslant\left|G_{1}\left(x_{1}, x\right)\right| / q^{(n-1)^{2}}$. For $1 \leqslant i \leqslant n$ let $Q(i)$ denote the center of $O_{p}\left(G\left(x_{i}\right)\right)$. If $Q(i) \leqslant G_{1}(x)$, then $Q(i) \leqslant G_{2}\left(x_{i}\right)=1$ since $Q(i) \& G\left(x_{i}\right)$. Since $O_{p}\left(G\left(x_{i}\right)\right) \neq 1, \quad Q(i) \nless G_{1}(x)$. Since $Q(i) \triangleleft G\left(x_{1}, x\right), \quad Q(i)^{\Gamma(x)} \geqslant$ $O_{p}\left(G\left(x_{i}, x\right)^{\Gamma(x)}\right)$ by the lemma. It follows that $\langle Q(1), \ldots, Q(n)\rangle$ acts transitively on $\Gamma(x)$. Since $J \leqslant O_{p}\left(G\left(x_{1}\right)\right) \cap \cdots \cap O_{p}\left(G\left(x_{n}\right)\right), J \leqslant G_{2}(x)=1$. It follows that $\left|G_{1}\left(x_{1}, x\right)\right| \mid q^{(n-1)^{2}}$.

\section{BIBLIOGRAPHY}

1. M. Bürker and W. Knapp, Zur Vermutung von Sims über primitive Permutationsgruppen II, Arch. Math. 27 (1976), 352-359.

2. H. M. S. Coxeter, Regular polytopes, 2nd ed., Macmillan, New York, 1963.

3. A. Gardiner, Arc transitivity in graphs, Quart. J. Math. Oxford 24 (1973), 399-407.

4. O. Schreier and B. L. van der Waerden, Die Automorphismen der projektiven Gruppen, Abh. Math. Sem. Univ. Hamburg 6 (1926), 321-346.

5. J. Tits, Buildings of spherical type and finite BN-pairs, Springer-Verlag, Berlin and New York, 1974.

6. W. T. Tutte, A family of cubical graphs, Proc. Cambridge Philos. Soc. 43 (1947), 459-474.

7. __ Connectivity in graphs, Univ. of Toronto Press, Toronto, 1966.

8. R. Weiss, Uber symmetrische Graphen und die projektiven Gruppen, Arch. Math. 28 (1977), $110-112$.

9. __ Symmetrische Graphen mit auflösbaren Stabilisatoren, J. Algebra. (to appear).

10. _ Groups with $a(B, N)$-pair and locally transitive graphs (submitted).

II. Mathematisches Institut, Freie Universität Berlin, D-1000 Berlin 33, Federal RePUblic OF GERMANY 